\title{
The impact of measurement error on principal component analysis
}

\author{
Running headline: Impact of measurement error on PCA
}

Kristoffer Hellton, Magne Thoresen

Department of Biostatistics, Institute of Basic Medical Sciences, University of Oslo

September 3, 2015 


\begin{abstract}
We investigate the effect of measurement error on principal component analysis in the high-dimensional setting. The effects of small random, additive errors are characterized by the expectation and variance of the changes in the eigenvalues and eigenvectors. The results show that the impact of uncorrelated measurement error on the principal component scores is mainly in terms of increased variability, with a relative small impact. In practice, this suggests that the impact will be negligible for the components corresponding to the largest eigenvalues, when these are used for visualizing data or in score-based classification and regression. However, the measurement error will contribute to a large variability in component loadings, relative to the loading values, such that interpretation based on the loadings can be difficult. The results are illustrated by simulating additive Gaussian measurement error in microarray expression data from cancer tumors and control tissues.
\end{abstract}

Keywords: High-dimensional data, eigenvalues, eigenvectors, measurement error, microarray, perturbation theory, principal component analysis.

\title{
1 Introduction
}

The last decades have seen an exploding production of complex, high-dimensional data in different fields, from genetics ( $\mathrm{Li} \& \mathrm{Xu}, 2008$ ) to finance (Fan et al., 2011). Often in these examples the sample size can be quite small compared to the number of measured variables, thus an efficient strategy for dimension reduction is required. Principal component analysis (PCA) is a widely used technique, which reduces the high-dimensional data to a small set of component scores. The component scores can be used for visualization and as input in conventional methods, such as classification, clustering and regression. In practice, the principal components are often thought to represent underlying processes, accounting for the variability in the data and the component loadings could be interpreted as the relative importance of the different variables in the unobserved processes.

In various high-dimensional data, we find that measurement error in the observed variables can be a severe problem. Examples include measurements of chemical spectra in chemometrics, fMRI brain scans or microarray expression data in genomics. In regression models, the presence of measurement error in covariates is known to cause bias in parameter estimates and loss of power to detect significant effects (Carroll et al., 2006; Buonaccorsi, 2009).

To deal with the issue of measurement error in PCA within the setting of microarrays and chemometrics, Sanguinetti et al. (2005) and Wentzell \& Hou (2012) constructed different variations of principal component analysis where information about the measurement error is incorporated. Wentzell \& Hou (2012) (based on Wentzell et al. (1997)) constructed a framework for maximum likelihood principal component analysis which incorporates an assumed known covariance matrix for the measurement error. Sanguinetti et al. (2005) extended the probabilistic PCA, which is solved by an EM-algorithm, to incorporate the technical precision connected to a microarray as a proxy for the measurement error in the data.

However, in practice it is difficult to estimate the covariance matrix of the measurement error in a high-dimensional situation, and the PCA versions accounting for measurement error are not in common use. Analyses are often carried out naively, running standard PCA on the observed data without any correction for measurement error and therefore it will be useful to understand the impact of error on component loadings, 
scores and selection. In the framework of chemometrics, the effect of measurement error on eigenvalues was investigated by Faber et al. (1993, 1995), but only for homogeneous error and not considering the high-dimensional situation.

In this paper, we will derive the bias and variability in loadings and scores caused by a general, additive measurement error. This is done by considering perturbations of the eigendecomposition, such that the bias and variability of the change in eigenvectors and -values are given by the distribution of the errors.

\section{Principal component analysis}

Principal component analysis reduces the dimensionality of data by finding the low-dimensional linear subspaces where the projections of the data have the largest possible variability. Specifically, given a $p$ dimensional vector $\mathbf{x}$, the first principal component is a unit-length vector $\mathbf{v}_{1} \in \mathcal{R}^{p}$, such that $\mathbf{v}_{1}^{T} \mathbf{x}$ has maximal variance. Since $\operatorname{Var} \mathbf{v}_{1}^{T} \mathbf{x}=\mathbf{v}_{1}^{T} \Sigma \mathbf{v}_{1}$, where $\Sigma$ is the population covariance matrix, the first principal component is the eigenvector corresponding to the largest eigenvalue. The second principal component $\mathbf{v}_{2}$ is the unit-length vector with the largest variance orthogonal to $\mathbf{v}_{1}$ and is given by the eigenvector corresponding to the second largest eigenvalue and so on.

In practice, the principal components are given by the sample covariance matrix. Let $\mathbf{X}_{1}, \ldots, \mathbf{X}_{n}$ be $n$ iid $p$-dimensional vectors and $\mathbf{X}=\left[\mathbf{X}_{1}, \ldots, \mathbf{X}_{n}\right]$ a $p \times n$ data matrix. When assuming for simplicity that $\mathbf{X}_{r}, r=1, \ldots, n$ has a known zero expectation, the sample covariance matrix is $\mathbf{S}_{X}=\frac{1}{n} \mathbf{X} \mathbf{X}^{T}$. The principal components are then given by the eigendecomposition of $\mathbf{S}_{X}$ given by

$$
\mathbf{S}_{X}=\mathbf{V} \boldsymbol{\Lambda} \mathbf{V}^{T}
$$

with eigenvalues $\boldsymbol{\Lambda}=\operatorname{diag}\left(\lambda_{1}, \ldots, \lambda_{p}\right)$ and eigenvectors $\mathbf{V}=\left[\mathbf{v}_{1}, \ldots, \mathbf{v}_{p}\right]$. The projections, denoted by $\mathbf{Z}_{i}=\mathbf{v}_{i}^{T} \mathbf{X}$ for $i=1, \ldots, p$, are referred to as the $i$ th scores and represent the new data which can be used in further analyses. As this linear projection can be seen as a weighted sum of the original variables, where the eigenvector gives the weight of each variable, the coefficients of the eigenvector are usually referred to as the loadings of the component. The dimensionality can be reduced by choosing the components corresponding to the largest eigenvalues to represent the data.

In a situation with measurement error, an error contaminated version of the data $\mathbf{W}$ is observed instead of the original data $\mathbf{X}$. For the classical, additive measurement error model, $\mathbf{X}$ is observed by $\mathbf{W}$ with the errors $\sigma \mathbf{U}$, such that

$$
\mathbf{W}=\mathbf{X}+\sigma \mathbf{U}
$$

The scaling $\sigma$ controls the magnitude of the error matrix $\mathbf{U}$. Both the data $\mathbf{X}$ and the error $\mathbf{U}$ are for simplicity assumed to have known zero expectation, hence the same is true for $\mathbf{W}$. Then the natural estimator for the population covariance matrix (unbiased in the classical case, $p<n$ ) is $\mathbf{S}_{W}=\frac{1}{n} \mathbf{W W}^{T}$. When the error model is additive, the covariance matrix of the observed data $\mathbf{W}$ is given by

$$
\frac{1}{n} \mathbf{W} \mathbf{W}^{T}=\frac{1}{n}(\mathbf{X}+\sigma \mathbf{U})(\mathbf{X}+\sigma \mathbf{U})^{T}=\frac{1}{n} \mathbf{X} \mathbf{X}^{T}+\frac{\sigma}{n} \mathbf{X} \mathbf{U}^{T}+\frac{\sigma}{n} \mathbf{U} \mathbf{X}^{T}+\frac{\sigma^{2}}{n} \mathbf{U U}^{T} .
$$

The covariance matrix $\mathbf{S}_{W}$ is decomposed into the covariance matrix $\mathbf{S}_{X}$ and the additive change depending 
on the scaling $\sigma$

$$
\mathbf{S}_{W}=\mathbf{S}_{X}+\sigma \Delta \mathbf{S}_{1}+\sigma^{2} \Delta \mathbf{S}_{2}
$$

where $\Delta \mathbf{S}_{1}=\frac{1}{n} \mathbf{X} \mathbf{U}^{T}+\frac{1}{n} \mathbf{U} \mathbf{X}^{T}$ and $\Delta \mathbf{S}_{2}=\frac{1}{n} \mathbf{U U}^{T}$.

Our aim is to assess the change in loadings, scores, and component selection, when the principal component analysis is carried out on $\mathbf{S}_{W}$ instead of $\mathbf{S}_{X}$. The eigenstructure of $\mathbf{S}_{X}$ is further assumed to be known in the sense that $\mathbf{X}$ is fixed. Then there will be $p$ fixed eigenvectors $\mathbf{V}=\left[\mathbf{v}_{1}, \ldots, \mathbf{v}_{p}\right]$ and eigenvalues $\boldsymbol{\Lambda}=\operatorname{diag}\left(\lambda_{1}, \ldots, \lambda_{p}\right)$. The eigenvalues are assumed to follow from a spiked covariance model, as introduced by Johnstone (2001), where the $m$ largest eigenvalues are substantially larger than the remaining $p-m$. In addition, as the data matrix $\mathbf{X}$ is fixed as a sample, the non-zero eigenvalues are necessarily different (Rao et al., 2008). Also as $p>n$ there must be at least $p-n$ zero eigenvalues, where we assume for simplicity that exactly $p-n$ eigenvalues are equal to zero, such that the eigenvalues of $\boldsymbol{\Lambda}$ fulfill

$$
\lambda_{1}>\cdots>\lambda_{m} \gg \lambda_{m+1}>\cdots>\lambda_{n}>\lambda_{n+1}=\cdots=\lambda_{p}=0
$$

\subsection{Perturbation problem}

Perturbation theory has been applied in several statistical settings, for instance by Kadane (1970) to investigate the effect of small errors on different estimators and restrictions for overidentification. Nadler (2008) used matrix perturbation theory to find finite sample approximations for estimates of the leading eigenvalue and eigenvector in a single-spike model. As the sample estimation error in PCA can be modeled as an independent homogeneous measurement error, the results of this paper will in this special case be similar to the results of Nadler (2008).

Our results have the following outline: First, the Taylor expansion of eigenvalues and eigenvectors of $\mathbf{S}_{W}$ are derived, giving the Taylor expansion of the principal component scores of $\mathbf{S}_{W}$. Then the expectation and variance of the difference between the eigenvectors and -values of $\mathbf{S}_{W}$ and $\mathbf{S}_{X}$ are derived based on the Taylor expansions. For these results, we condition on the original data matrix $\mathbf{X}$, such that $\mathbf{X}$ represents $n$ fixed realizations from a population distribution. The Taylor expansions of eigenvalues and eigenvectors have earlier been investigated by Wilkinson (1965) and Stewart \& Sun (1990) for deterministic matrices in numerical perturbation analysis, while Stewart (1990) introduced a stochastic norm which also allows random error matrices. We denote the $i$ th eigenvalues and -vectors of $\mathbf{S}_{X}$ by $\lambda_{i}$ and $\mathbf{v}_{i}$ and the $i$ th eigenvalues and -vectors of $\mathbf{S}_{W}$ by $\lambda_{W, i}$ and $\mathbf{v}_{W, i}$ :

Lemma 1. Assuming fixed $p \times n$ matrices $\mathbf{X}$ and $\mathbf{U}$, the Taylor expansion for the ith eigenvalue of $\mathbf{S}_{W}$ as $\sigma \rightarrow 0$ is given by

$$
\lambda_{W, i}=\lambda_{i}+\sigma \mathbf{v}_{i}^{T} \Delta \mathbf{S}_{1} \mathbf{v}_{i}+\sigma^{2} \mathbf{v}_{i}^{T} \Delta \mathbf{S}_{2} \mathbf{v}_{i}+\sigma^{2} \sum_{j \neq i} \frac{\mathbf{v}_{i}^{T} \Delta \mathbf{S}_{1} \mathbf{v}_{j} \mathbf{v}_{j}^{T} \Delta \mathbf{S}_{1} \mathbf{v}_{i}}{\lambda_{i}-\lambda_{j}}+O\left(\sigma^{3}\right),
$$


and the Taylor expansion for the ith eigenvector of $\mathbf{S}_{W}$ up to a scaling constant as $\sigma \rightarrow 0$ is given by

$$
\begin{aligned}
\mathbf{v}_{W, i} & =\mathbf{v}_{i}+\sigma \sum_{j \neq i} \frac{\mathbf{v}_{j}^{T} \Delta \mathbf{S}_{1} \mathbf{v}_{i}}{\lambda_{i}-\lambda_{j}} \mathbf{v}_{j}+\sigma^{2} \sum_{j \neq i} \frac{\mathbf{v}_{j}^{T} \Delta \mathbf{S}_{2} \mathbf{v}_{i}}{\lambda_{i}-\lambda_{j}} \mathbf{v}_{j} \\
& +\sigma^{2} \sum_{j \neq i} \sum_{k \neq i} \frac{\mathbf{v}_{j}^{T} \Delta \mathbf{S}_{1} \mathbf{v}_{i} \mathbf{v}_{j}^{T} \Delta \mathbf{S}_{1} \mathbf{v}_{k}}{\left(\lambda_{i}-\lambda_{j}\right)\left(\lambda_{i}-\lambda_{k}\right)} \mathbf{v}_{j}-\sigma^{2} \sum_{j \neq i} \frac{\mathbf{v}_{j}^{T} \Delta \mathbf{S}_{1} \mathbf{v}_{i} \mathbf{v}_{i}^{T} \Delta \mathbf{S}_{1} \mathbf{v}_{i}}{\left(\lambda_{i}-\lambda_{j}\right)^{2}} \mathbf{v}_{j}+O\left(\sigma^{3}\right),
\end{aligned}
$$

where $i=1, \ldots, p$ when $p \leq n$ and $i=1, \ldots, n$ when $p>n$. The proof is found in Appendix A.1.

The first theorem establishes the Taylor expansion of the principal component scores. We denote the $i$ th scores of $\mathbf{S}_{X}$ by $\mathbf{Z}_{i}$ and the $i$ th scores of $\mathbf{S}_{W}$ by $\mathbf{Z}_{W, i}$. As the scores are given by $\mathbf{Z}_{W, i}=\mathbf{v}_{W, i}^{T} \mathbf{W}$, the result follows from the Taylor expansion of the eigenvectors combined with the observed data matrix $\mathbf{W}$.

Theorem 1. Assuming fixed $p \times n$ matrices $\mathbf{X}$ and $\mathbf{U}$, the Taylor expansion for the ith component scores of $\mathbf{S}_{W}$ as $\sigma \rightarrow 0$ is given by

$$
\begin{aligned}
\mathbf{Z}_{W, i} & =\mathbf{Z}_{i}+\sigma \sum_{j \neq i} \frac{\mathbf{v}_{j}^{T} \Delta \mathbf{S}_{1} \mathbf{v}_{i}}{\lambda_{i}-\lambda_{j}} \mathbf{v}_{j}^{T} \mathbf{X}+\sigma \mathbf{v}_{i}^{T} \mathbf{U}+\sigma^{2} \sum_{j \neq i} \frac{\mathbf{v}_{j}^{T} \Delta \mathbf{S}_{1} \mathbf{v}_{i}}{\lambda_{i}-\lambda_{j}} \mathbf{v}_{j}^{T} \mathbf{U}+\sigma^{2} \sum_{j \neq i} \frac{\mathbf{v}_{j}^{T} \Delta \mathbf{S}_{2} \mathbf{v}_{i}}{\lambda_{i}-\lambda_{j}} \mathbf{v}_{j}^{T} \mathbf{X} \\
& +\sigma^{2} \sum_{j \neq i} \sum_{k \neq i} \frac{\mathbf{v}_{j}^{T} \Delta \mathbf{S}_{1} \mathbf{v}_{i} \mathbf{v}_{j}^{T} \Delta \mathbf{S}_{1} \mathbf{v}_{k}}{\left(\lambda_{i}-\lambda_{j}\right)\left(\lambda_{i}-\lambda_{k}\right)} \mathbf{v}_{j}^{T} \mathbf{X}-\sigma^{2} \sum_{j \neq i} \frac{\mathbf{v}_{j}^{T} \Delta \mathbf{S}_{1} \mathbf{v}_{i} \mathbf{v}_{i}^{T} \Delta \mathbf{S}_{1} \mathbf{v}_{i}}{\left(\lambda_{i}-\lambda_{j}\right)^{2}} \mathbf{v}_{j}^{T} \mathbf{X}+O\left(\sigma^{4}\right),
\end{aligned}
$$

where $i=1, \ldots, p$ when $p \leq n$ and $i=1, \ldots, n$ when $p>n$. The proof is found in Appendix A.2.

The change induced by the measurement error can be quantified by the difference between the eigenvalues and eigenvectors of $\mathbf{S}_{W}$ and $\mathbf{S}_{X}$, denoted by $\Delta \lambda_{i}$ and $\Delta \mathbf{v}_{i}$ :

$$
\Delta \lambda_{i}=\lambda_{W, i}-\lambda_{i}, \quad \Delta \mathbf{v}_{i}=\mathbf{v}_{W, i}-\mathbf{v}_{i} .
$$

We use the results in Theorem 1 and Lemma 1 to derive the expectation and variability of $\Delta \lambda_{W, i}$ and $\Delta \mathbf{v}_{W, i}$ under the assumption that $\sigma \mathbf{U}$ are normally distributed and $\mathbf{X}_{r}$ and $\mathbf{U}_{r}$ are independent. Then the multivariate additive measurement error model for $n$ samples $\mathbf{W}=\left[\mathbf{W}_{1}, \cdots, \mathbf{W}_{n}\right]$ is given by

$$
\mathbf{W}_{r}=\mathbf{X}_{r}+\sigma \mathbf{U}_{r}, \quad \mathbf{U}_{r} \sim \mathcal{N}\left(\mathbf{0}, \Sigma_{U}\right), \quad r=1, \ldots, n
$$

The covariance matrix of the error $\sigma \mathbf{U}_{r}$ is given $\operatorname{Var}\left(\sigma \mathbf{U}_{r}\right)=\sigma^{2} \Sigma_{U}$, such that $\sigma^{2}$ controls the scaling of the variance. The expectation of $\Delta \lambda_{i}$ and $\Delta \mathbf{v}_{i}$ give the bias in $\lambda_{W, i}$ and $\mathbf{v}_{W, i}$.

Theorem 2 (Eigenvalues and eigenvectors). If $\mathbf{U}=\left[\mathbf{U}_{1}, \ldots, \mathbf{U}_{n}\right]$ is independent, identically, normally distributed, $\mathbf{U}_{r} \sim \mathcal{N}\left(\mathbf{0}, \Sigma_{U}\right)$ for $r=1, \ldots, n$, the expectation and variance of $\Delta \lambda_{i}$ as $\sigma \rightarrow 0$, conditional on $\mathbf{X}$, are given by

$$
\begin{aligned}
\mathbb{E}\left(\Delta \lambda_{i} \mid \mathbf{X}\right) & =\sigma^{2} \mathbf{v}_{i}^{T} \Sigma_{U} \mathbf{v}_{i}+\frac{\sigma^{2}}{n} \sum_{j \neq i} \frac{\lambda_{i} \mathbf{v}_{j}^{T} \Sigma_{U} \mathbf{v}_{j}+\lambda_{j} \mathbf{v}_{i}^{T} \Sigma_{U} \mathbf{v}_{i}}{\lambda_{i}-\lambda_{j}}+O\left(\sigma^{3}\right), \\
\operatorname{Var}\left(\Delta \lambda_{i} \mid \mathbf{X}\right) & =\frac{4 \lambda_{i} \sigma^{2}}{n} \mathbf{v}_{i}^{T} \Sigma_{U} \mathbf{v}_{i}+O\left(\sigma^{3}\right) .
\end{aligned}
$$


The expectation of $\Delta \mathbf{v}_{i}$ as $\sigma \rightarrow 0$, conditional on $\mathbf{X}$, is given by

$$
\mathbb{E}\left(\Delta \mathbf{v}_{i} \mid \mathbf{X}\right)=\sigma^{2} \sum_{j \neq i} \frac{\mathbf{v}_{j}^{T} \Sigma_{U} \mathbf{v}_{i}}{\lambda_{i}-\lambda_{j}} \mathbf{v}_{j}-\frac{\sigma^{2}}{n} \sum_{j \neq i} \frac{2 \mathbf{v}_{j}^{T} \Sigma_{U} \mathbf{v}_{i}}{\lambda_{i}-\lambda_{j}} \mathbf{v}_{j}+\frac{\sigma^{2}}{n} \sum_{j \neq i} \sum_{k \neq i, j} \frac{\lambda_{j} \mathbf{v}_{k}^{T} \Sigma_{U} \mathbf{v}_{i}}{\left(\lambda_{i}-\lambda_{j}\right)\left(\lambda_{i}-\lambda_{k}\right)} \mathbf{v}_{j}+O\left(\sigma^{3}\right),
$$

and the variance of the $k$ th coefficient of $\Delta \mathbf{v}_{i}$ is given by

$$
\operatorname{Var}\left(\Delta \mathbf{v}_{i k} \mid \mathbf{X}\right)=\frac{\sigma^{2}}{n} \sum_{j \neq i} \frac{\lambda_{j} \mathbf{v}_{i}^{T} \Sigma_{U} \mathbf{v}_{i}+\lambda_{i} \mathbf{v}_{j}^{T} \Sigma_{U} \mathbf{v}_{j}}{\left(\lambda_{i}-\lambda_{j}\right)^{2}} \mathbf{v}_{j k}^{2}+\frac{\sigma^{2}}{n} \sum_{j, l \neq i, j<l} \frac{2 \lambda_{i} \mathbf{v}_{j}^{T} \Sigma_{U} \mathbf{v}_{l}}{\left(\lambda_{i}-\lambda_{j}\right)\left(\lambda_{i}-\lambda_{l}\right)} \mathbf{v}_{j k} \mathbf{v}_{l k}+O\left(\sigma^{4}\right),
$$

where $i=1, \ldots, p$ when $p \leq n$ and $i=1, \ldots, n$ when $p>n$. The proof is found in Appendix A.4.

The variance of $\Delta \mathbf{v}_{i k}$ is, to leading order, a weighted sum over the $k$ th coordinate of all other eigenvectors, where the weights depend on the data and error structure through the eigenvalues and the covariance matrix of the error.

Theorem 3 (Scores). If $\mathbf{U}=\left[\mathbf{U}_{1}, \ldots, \mathbf{U}_{n}\right]$ is independent, identically, normally distributed, $\mathbf{U}_{r} \sim \mathcal{N}\left(\mathbf{0}, \Sigma_{U}\right)$ for $r=1, \ldots, n$, the expectation of $\Delta \mathbf{Z}_{i}=\mathbf{Z}_{W, i}-\mathbf{Z}_{i}$ as $\sigma \rightarrow 0$, conditional on $\mathbf{X}$, is given by

$$
\begin{aligned}
\mathbb{E}\left(\Delta \mathbf{Z}_{i} \mid \mathbf{X}\right) & =\sigma^{2} \sum_{j \neq i} \frac{\mathbf{v}_{j}^{T} \Sigma_{U} \mathbf{v}_{i}}{\lambda_{i}-\lambda_{j}} \mathbf{v}_{j}^{T} \mathbf{X}-\frac{\sigma^{2}}{n} \sum_{j \neq i} \frac{2 \mathbf{v}_{j}^{T} \Sigma_{U} \mathbf{v}_{i}}{\lambda_{i}-\lambda_{j}} \mathbf{v}_{j}^{T} \mathbf{X}+\frac{\sigma^{2}}{n} \sum_{j \neq i} \sum_{k \neq i, j} \frac{\lambda_{j} \mathbf{v}_{k}^{T} \Sigma_{U} \mathbf{v}_{i}}{\left(\lambda_{i}-\lambda_{j}\right)\left(\lambda_{i}-\lambda_{k}\right)} \mathbf{v}_{j}^{T} \mathbf{X} \\
& +\frac{\sigma^{2}}{n} \sum_{j \neq i} \frac{\mathbf{v}_{j}^{T} \Sigma_{U} \mathbf{v}_{i} \mathbf{v}_{j}^{T} \mathbf{X}+\mathbf{v}_{j}^{T} \Sigma_{U} \mathbf{v}_{i} \mathbf{v}_{i}^{T} \mathbf{X}}{\lambda_{i}-\lambda_{j}}+O\left(\sigma^{3}\right) .
\end{aligned}
$$

The variance of the $k$ th coefficient of $\Delta \mathbf{Z}_{i}$ as $\sigma \rightarrow 0$, conditional on $\mathbf{X}$, is given by

$$
\begin{aligned}
\operatorname{Var}\left(\Delta \mathbf{Z}_{i k} \mid \mathbf{X}\right) & =\sigma^{2} \mathbf{v}_{i}^{T} \Sigma_{U} \mathbf{v}_{i}+\frac{\sigma^{2}}{n} \sum_{j \neq i} \frac{\lambda_{j} \mathbf{v}_{i}^{T} \Sigma_{U} \mathbf{v}_{i}+\lambda_{i} \mathbf{v}_{j}^{T} \Sigma_{U} \mathbf{v}_{j}}{\left(\lambda_{i}-\lambda_{j}\right)^{2}}\left(\mathbf{v}_{j}^{T} \mathbf{X}_{k}\right)^{2} \\
& +\frac{\sigma^{2}}{n} \sum_{j, l \neq i, j<l} \frac{2 \lambda_{i} \mathbf{v}_{j}^{T} \Sigma_{U} \mathbf{v}_{l} \mathbf{v}_{j}^{T} \mathbf{X}_{k} \mathbf{v}_{l}^{T} \mathbf{X}_{k}}{\left(\lambda_{i}-\lambda_{j}\right)\left(\lambda_{i}-\lambda_{l}\right)}+\frac{2 \sigma^{2}}{n} \sum_{j \neq i} \frac{\mathbf{v}_{j}^{T} \Sigma_{U} \mathbf{v}_{i} \mathbf{v}_{i}^{T} \mathbf{X}_{k}+\mathbf{v}_{i}^{T} \Sigma_{U} \mathbf{v}_{i} \mathbf{v}_{j}^{T} \mathbf{X}_{k}}{\lambda_{i}-\lambda_{j}} \mathbf{v}_{j} \mathbf{X}_{k}+O\left(\sigma^{4}\right),
\end{aligned}
$$

where $i=1, \ldots, p$ when $p \leq n$ and $i=1, \ldots, n$ when $p>n$. The proof is found in Appendix A.5.

Remark 1. If the measurement error is homogeneous, the bias in the eigenvalues, eigenvectors and scores simplifies. As $\mathbf{U}_{r} \sim N\left(0, \sigma^{2} I\right)$ for $r=1, \ldots, n, \mathbb{E}\left(\mathbf{S}_{W} \mid \mathbf{X}\right)=\mathbf{S}_{X}+\sigma^{2} I$. Therefore $\lambda_{W, i}=\lambda_{i}+\sigma^{2}$ and $\mathbf{v}_{W, i}=\mathbf{v}_{i}$ and the bias is exactly given

$$
\mathbb{E}\left(\Delta \lambda_{i} \mid \mathbf{X}\right)=\sigma^{2}, \quad \mathbb{E}\left(\Delta \mathbf{v}_{i} \mid \mathbf{X}\right)=\mathbf{0}
$$

\section{Implications}

We will now explore the implications of Theorems 2 and 3 for the loadings, scores and component selection, when the measurement error is assumed to be uncorrelated and either homogeneous or heterogeneous. In the case of independent, homogeneous measurement error, the variance of $\mathbf{U}_{r}$ is equal for all variables, 
such that $\Sigma_{U}=I$. Then the covariance matrix of the error is given as $\operatorname{Var}\left(\sigma \mathbf{U}_{r}\right)=\sigma^{2} I$. In the case of independent, heterogeneous measurement error, the variance of $\mathbf{U}_{r}$ is different in each variable, such that $\Sigma_{U}=\operatorname{diag}\left(c_{1}, \ldots, c_{p}\right)$, where the constants $c_{k}$ give the relative size of the variances. Then the covariance matrix of the error is $\operatorname{Var}\left(\sigma \mathbf{U}_{r}\right)=\operatorname{diag}\left(\sigma^{2} c_{1}, \ldots, \sigma^{2} c_{p}\right)$, where $\sigma^{2}$ controls the scaling.

A key element in the bias and variance expressions of Theorem 2 and 3 is the quantity $\mathbf{v}_{j}^{T} \Sigma_{U} \mathbf{v}_{i}$, which captures the relationship between the error and the original data. For $j=i$, this corresponds to a projection of the error covariance matrix $\Sigma_{U}$ onto the eigenvector space spanned by $\mathbf{v}_{i}$. For independent, homogeneous error, the projection of $\Sigma_{U}$ is either $\mathbf{v}_{i}^{T} \Sigma_{U} \mathbf{v}_{i}=1$ and $\mathbf{v}_{j}^{T} \Sigma_{U} \mathbf{v}_{i}=0$ for $j \neq i$, which simplify the bias and variance expressions. For independent, heterogeneous error with covariance matrix $\Sigma_{U}=\operatorname{diag}\left(c_{1}, \ldots, c_{p}\right)$, the projections are given as weighted sums $\mathbf{v}_{i}^{T} \Sigma_{U} \mathbf{v}_{i}=\sum_{k=1}^{p} c_{k} \mathbf{v}_{k i}^{2}$ and $\mathbf{v}_{j}^{T} \Sigma_{U} \mathbf{v}_{i}=\sum_{k=1}^{p} c_{k} \mathbf{v}_{k j} \mathbf{v}_{k i}$, where the variances are weighted by the corresponding loadings. Since the sum of the weights $\mathbf{v}_{k i}^{2}$ are normalized to 1 , the $\mathbf{v}_{i}^{T} \Sigma_{U} \mathbf{v}_{i}$ will be a weighted average of the error variances in the heterogeneous case.

\subsection{Loadings}

The impact of measurement error on the principal component loadings can be assessed through the bias and variance in the eigenvectors. If the measurement error structure is independent and homogeneous, the bias in the loadings will be zero due to the orthogonality of the eigenvectors. However, heterogeneous error or error structures with dependencies will introduce a bias.

We can illustrate this effect through a simple heterogeneous structure with measurement error in only one variable, $\Sigma_{U}=\operatorname{diag}(1,0, \ldots, 0)$. The bias in the first loading of the $i$ th component is given by

$$
\mathbb{E}\left(\Delta \mathbf{v}_{i 1} \mid \mathbf{X}\right)=\sigma^{2} \mathbf{v}_{i 1}\left(1-\frac{2}{n}\right) \sum_{j \neq i} \frac{\mathbf{v}_{i j}^{2}}{\lambda_{i}-\lambda_{j}}+\frac{\sigma^{2} \mathbf{v}_{i 1}}{n} \sum_{j \neq i} \sum_{k \neq i, j} \frac{\lambda_{j} \mathbf{v}_{i j} \mathbf{v}_{i k}}{\left(\lambda_{i}-\lambda_{j}\right)\left(\lambda_{i}-\lambda_{k}\right)}+O\left(\sigma^{3}\right),
$$

As $p>n$, we have assumed the fixed eigenvalues to be zero for $j=n+1, \ldots, p$. If $p$ is much larger then $n$, the first two sums in (12) are approximated by $\left(1 / \lambda_{i}\right) \sum_{j \neq i} \mathbf{v}_{i j}^{2}$ and due to the unit-length of the eigenvectors, we have $\sum_{j \neq i} \mathbf{v}_{i j}^{2} \simeq 1$, such that expression (12) is approximately given by

$$
\mathbb{E}\left(\Delta \mathbf{v}_{i 1} \mid \mathbf{X}\right) \simeq \frac{\sigma^{2}}{\lambda_{i}} \mathbf{v}_{i 1}
$$

The bias in the first loading in this simplified model depends, to leading order, on the loading value itself, thus the larger loadings have larger bias. It also depends on $\sigma^{2} / \lambda_{i}$, the ratio between the variance of the error $\sigma \mathbf{U}$ and the $i$ th eigenvalue of $\mathbf{S}_{X}$. This ratio expresses an inverse signal-to-noise relationship, as the eigenvalues represent the overall structure or signal in the data. When the eigenvalues are large compared to the error variance, the inverse signal-to-noise ratio is close to zero, resulting in a very small bias in the eigenvector coefficients. The ratio $\sigma^{2} / \lambda_{i}$ is always positive, such that the loading corresponding to the variable with increased variability is overestimated in absolute value and thereby also the importance of the variable in question. This is natural as principal component analysis is constructed to interpret high variability as important structure. As errors increase variability, the variables affected by error will erroneously be assigned an increased importance. For a general independent, heterogeneous error structure, i.e. $\Sigma_{U}=\operatorname{diag}\left(c_{1}, \ldots, c_{p}\right)$, the bias will depend on whether the corresponding error variance $\sigma^{2} c_{k}$ is smaller 
or larger than the average error variance over all variables:

$$
\mathbb{E}\left(\Delta \mathbf{v}_{i k} \mid \mathbf{X}\right) \simeq \frac{\sigma^{2}\left(c_{k}-\bar{c}\right)}{\lambda_{i}} \mathbf{v}_{i k}
$$

where $\bar{c}=(1 / p) \sum_{i=1}^{p} c_{p}$ is the mean of individual variances.

The variation in a loading is characterized by $\operatorname{Var}\left(\Delta \mathbf{v}_{i k} \mid \mathbf{X}\right)$ in equation (9). If the error is independent and homogeneous, the variance is given by

$$
\operatorname{Var}\left(\Delta \mathbf{v}_{i k} \mid \mathbf{X}\right)=\frac{\sigma^{2}}{n} \sum_{j \neq i} \frac{\lambda_{i}+\lambda_{j}}{\left(\lambda_{i}-\lambda_{j}\right)^{2}} \mathbf{v}_{j k}^{2}+O\left(\sigma^{4}\right) .
$$

The variance is, to leading order, a weighted sum of the eigenvalues, where the weights $\mathbf{v}_{j k}^{2}$ are the $k$ th square coefficient of all other eigenvectors and this makes it difficult to assess the magnitude of the variance. But as the average value of $\mathbf{v}_{j k}^{2}$ is $1 / p$ by construction, we have approximately $\sum_{j \neq i} \mathbf{v}_{j k}^{2} \simeq 1$. When $p \gg n$ and most eigenvalues are zero, the sum in (13) can be approximated $\sum_{j \neq i} \frac{\lambda_{i}+\lambda_{j}}{\left(\lambda_{i}-\lambda_{j}\right)^{2}} \mathbf{v}_{j k}^{2} \simeq\left(1 / \lambda_{i}\right) \sum_{j \neq i} \mathbf{v}_{i j}^{2}$. Therefore we can approximate the variability in each loading of the $i$ th component by

$$
\operatorname{Var}\left(\Delta \mathbf{v}_{i k} \mid \mathbf{X}\right) \simeq \frac{\sigma^{2}}{\lambda_{i}} \frac{1}{n}
$$

The variability of the loadings within the same component will therefore be of the same magnitude and the variation should be seen relative to the loading value. From the example presented in Section 4, we will see that the variation will be small compared to the largest loadings, but large enough to be problematic for the average or small loadings. The large variability around the true value may cause the interpretation based on the loadings to be incorrect.

\subsection{Scores}

The projections of the original data onto the eigenvector space, the component scores, are often used in other types of analyses, such that the measurement error is propagated further. In the case of independent and homogeneous error, $\Sigma_{U}=I$, the bias in the scores will be zero,

$$
\mathbb{E}\left(\Delta \mathbf{Z}_{i k} \mid \mathbf{X}\right)=\left(\sigma^{3}\right)
$$

while the variance in $k$ th score of the $i$ th component is given by

$$
\operatorname{Var}\left(\Delta \mathbf{Z}_{i k} \mid \mathbf{X}\right)=\sigma^{2}+\frac{\sigma^{2}}{n} \sum_{j \neq i} \frac{3 \lambda_{i}-\lambda_{j}}{\left(\lambda_{i}-\lambda_{j}\right)^{2}}\left(\mathbf{v}_{j}^{T} \mathbf{X}_{k}\right)^{2}+O\left(\sigma^{4}\right)
$$

by collecting the second and last term in (11). The first term in the variance expression is the largest, such that the variance in the scores is mainly given by the error term $\sigma \mathbf{U}$. It is however difficult to assess the contribution of the second term without the specified scores. The variability in scores can be compared to the variability in the observed data $\mathbf{W}$, which is given by the error $\operatorname{Var}\left(\mathbf{U}_{r k} \mid \mathbf{X}\right)=\sigma^{2}$. We see that the error variance in the scores is larger, due to the erroneously estimated eigenvectors. 
It is also possible to quantify the impact of the error in terms of the overall variance of the scores, as this is given by the eigenvalues $\operatorname{Var}(\mathbf{Z})=\Lambda$. The difference in the overall score variability is given by the bias in the eigenvalues, which for a homogeneous error is given by

$$
\mathbb{E}\left(\Delta \lambda_{i} \mid \mathbf{X}\right)=\sigma^{2}+\frac{\sigma^{2}}{n} \sum_{j \neq i} \frac{\lambda_{i}+\lambda_{j}}{\lambda_{i}-\lambda_{j}}+O\left(\sigma^{3}\right) .
$$

This expression can, when $p \gg n$, by approximated by $\mathbb{E}\left(\Delta \lambda_{i} \mid \mathbf{X}\right) \simeq \sigma^{2}\left(1+\frac{p}{n}\right)$, in the case of independent and homogeneous error. To assess the relative increase in the variance of the component scores, we compare the bias in the eigenvalues to the original eigenvalues, $\lambda_{i}$. If the eigenvalues are large, the relative increase in variability introduced by the error will be small.

\subsection{Component selection}

Dimension reduction can be achieved by selecting a subset of the components with the largest eigenvalues. Ferré (1995) performed an extensive comparison of different selection methods and concluded that there is no ideal selection criterion. However the criteria most often used in practice, the percentage rule, the Kaiser rule and Scree plot, all specify a cut-off based on the eigenvalues where only the components corresponding to the eigenvalues above the cut-off value are kept.

According to the percentage rule, the chosen components will explain a specified proportion of the total data variability. Since the eigenvalues give the variance of the components, the proportion is given by the sum of the eigenvalues of the chosen components divided by the sum of all eigenvalues. As the bias in the eigenvalues is approximately equal when $p \gg n$, the relative difference between the large and the small eigenvalues becomes smaller, such that additional components are needed to explain the same proportion of variability. The eigenvalues of the additional components must outweigh the difference between the sum of the bias in the chosen eigenvalues and the sum of the bias in all eigenvalues. The fact that additional components must be chosen is a result of the error obscuring the original data structure.

With the Kaiser rule, the cut-off is the mean of the eigenvalues $\bar{\lambda}$ (Jolliffe, 2002). Simulations show that too few variables will be selected under this rule and Jolliffe (2002) suggested a modified Kaiser rule with $0.7 \bar{\lambda}$ as the cut-off. The Kaiser rule will, as opposed to the percentage rule, adapt to the introduced bias. If the bias is approximately equal in all eigenvalues, the increase in $\bar{\lambda}$ will be the same as in the individual eigenvalues, such that the number of components over the cut-off value remains the same.

A Scree plot is a graphical procedure to determine a cut-off value, where the eigenvalues are plotted in decreasing order. The cut-off is set where the slope of the eigenvalues shifts from steep to shallow (Jolliffe, 2002) and the components above this breakpoint are retained. As the bias in the eigenvalues is approximately equal, the break point should not appear to move, but a graphical procedure can be difficult to assess.

\section{Example - Microarray expression data}

We illustrate our results with microarray expression data from lung cancer patients available in the ArrayExpress database (www.ebi.ac.uk/arrayexpress) under accession number E-GEOD-10072. The data set consists of 107 samples in total with 58 adenocarcinoma tumor tissue samples and 49 non-tumor samples. 
In all samples, 22284 genes are analyzed using a HG-U133A Affymetrix GeneChip.

Research into measurement error in microarray expression data has suggested a combination of additive and multiplicative errors (Rocke \& Durbin, 2001; Karakach \& Wentzell, 2007). For the purpose of illustration, we will only assume an additive measurement error. With the Affymetrix chip technology, it is possible to use probe information to estimate the technical uncertainty in expression values, for instance by the Bayesian Gene Expression (BGX) methodology (Hein et al., 2005; Turro et al., 2007). BGX uses Bayesian hierarchical models to produce a posteriori distributions of the gene expressions by utilizing probe information. The probe set in an Affymetrix GeneChip consists of 11 to 20 probe pairs of perfect match probes and mismatch probes, which accounts for different sources of noise (Hein et al., 2005). The method supplies a posteriori distributions for two parameters, the gene expression $\mu_{k}$ and the technical variability $\sigma_{k}^{2}$ for each sample.

We use the mean of the a posteriori distribution of $\mu_{k}$ as an estimate for the $k$ th gene expression and we use the mean of the distribution of $\sigma_{k}^{2}$ as an estimate of the gene- and sample-specific technical variance. However, we assume the technical variability to be equal for each sample and use the mean over all samples as our estimate of the measurement error.

The R package for BGX is highly labor-intensive and our analysis is restricted to the 3000 genes with the highest variance. The estimated gene expression is seen as the original data and the measurement error structure is assumed to be independent and heterogeneous with variance equal to the mean of the estimated technical variability over samples. To illustrate the effects of measurement error, we add a simulated Gaussian error to the data and the principal components of the original and the error-prone data are compared. The robustness of the component loadings against error is explored with the aim of biological interpretation, while the robustness of component scores is explored with the aim of classification and logistic regression.

The simulated additive measurement error is assumed to be normally distributed, with an independent and heterogeneous variance structure given by

$$
\mathbf{W}=\mathbf{X}+\mathbf{U}, \quad U_{r k} \stackrel{i i d}{\sim} \mathcal{N}\left(0, \sigma_{k}^{2}\right), \quad r=1, \ldots, n, k=1, \ldots, p,
$$

where the error variance $\sigma_{k}^{2}$ is the estimate supplied by the BGX methodology.

[Table 1 here.]

Table 1 displays the fifteen genes corresponding to the largest loadings (in absolute value) in the first principal component. The second column displays the original loadings and the third column displays the difference in the loadings, when the simulated error is added to the data. The fourth and fifth column display the theoretical bias and standard deviation in each loading. The two last columns display the genetic variance in the original data and the ratio between the measurement variance estimated by BGX and the genetic variance. The last column therefore shows the degree of uncertainty in the measurements.

We observe that changes in the loadings in Table 1 are much larger than the theoretical bias, and this is due to the variability in the scores. The theoretical variability, in terms of the standard deviation, is substantially larger than the theoretical bias, as seen in Table 1. This illustrates that, when focusing on the loadings, the main impact of uncorrelated errors is increased variability and not bias. Biologically, the loadings can be interpreted as the relative importance of each gene in the underlying processes represented by the component, and the random fluctuations in the loading values can undermine the biological interpretation. 
The impact of measurement error on the scores is illustrated graphically in Figure 1, which displays the first and second principal component of the original data and the data with a simulated, additive error. An arrow indicates the change in scores, when the simulated error is introduced. We observe that the changes are very small compared to the overall positions of the the scores, and this is due to the large first and second eigenvalues, $\lambda_{1}=1255.01$ and $\lambda_{2}=969.95$. The variance of error ranges from 0.03 to 1.90 with a mean of 0.86. Even though the error variance can be substantial compared to the genetic variability, it is very small compared to the two first eigenvalues, and the relative impact of the error on the scores is determined by the ratio between the error variance and the eigenvalue. The plot of the first two components can be used to classify the tissues by cancer status, adenocarcinoma tumor or non-tumor, which are indicated by black and open circles, respectively. The arrows illustrate that both groups experience a slightly increased variability. This is only a problem for classification if the change causes the groups to overlap, but this does not occur in our example. The key point is the small relative change in the overall positions of the scores.

[Figure 1 here]

The classification can also be done by logistic regression, where measurement error will often cause attenuation in estimated regression coefficients (Carroll et al., 2006; Buonaccorsi, 2009). We illustrate this effect by using the first component scores $\mathbf{Z}_{1}$ without and with error in a logistic regression. For logistic regression, we assume

$$
y_{i} \sim \operatorname{Bernoulli}\left(p_{i}\right), \quad \operatorname{logit}\left(p_{i}\right)=\beta_{0}+\beta_{1} \mathbf{Z}_{1} .
$$

The binary outcome $y_{i}$ is the cancer status, lung cancer or normal tissue. The estimated coefficients from the logistic regression based on the scores from the original data are $\hat{\beta}_{0, \mathbf{Z}_{1}}=-0.292$ and $\hat{\beta}_{1, \mathbf{Z}_{1}}=-7.224 \cdot 10^{-2}$,

while the coefficients based on the scores from the data with error are $\hat{\beta}_{0, \mathbf{Z}_{W, 1}}=-0.287$ and $\hat{\beta}_{1, \mathbf{Z}_{W, 1}}=$ $-7.161 \cdot 10^{-2}$. There is a slight under-estimation of the slope coefficient, consistent with the well-known attenuation effect. The increased variability in the component scores causes the estimated slope $\beta_{1, \mathbf{Z}_{W, 1}}$ to decrease in absolute value. The attenuation factor gives the expected decrease as $\beta_{1, \mathbf{Z}_{W, 1}}=\psi \beta_{1, \mathbf{Z}_{1}}$ with $\psi=\operatorname{Var} \mathbf{Z}_{1} / \operatorname{Var} \mathbf{Z}_{W, 1}$, (Carroll et al., 2006). As the variances of the scores are the eigenvalues, the factor is approximately $\lambda_{1} / \lambda_{1, W}=0.981$ in our data, consistent with the effect seen in $\beta_{1, \mathbf{Z}_{W, 1}}$.

\section{Discussion}

Our aim is to understand the effect of measurement error on principal component analysis, motivated by applications in high-dimensional error-prone data. The impact of the error is characterized by the bias and variance of eigenvalues and - vectors based on second-order Taylor approximations. The results are given for additive errors with a general covariance matrix, such that also measurement error with a correlation structure beyond the independent case can be explored. It has been shown that the impact of independent errors on component scores will be mainly in terms of an increased variability. We have quantified the impact of the additive measurement error based on a small error assumption. In practice, what we need for the theory to work is that $\sigma$ is small relative to the eigenvalues. As shown in the example, this will often be the case, even if there is substantial measurement error relative to the variation in the data themselves. In the setting of microarray data, where the first eigenvalues can be substantially larger than the error variance, the relative impact of the error variability will be negligible. This suggests that the additive measurement 
error might be unproblematic in microarrays, when dealing only with the components corresponding to the largest eigenvalues, for instance in the case of data visualization. However, the measurement error will also cause an increased variability in the loadings, which can be large relative to the loading values and thereby undermine their interpretation.

For the specific application of microarray data, the effects of multiplicative error should also be investigated, as Rocke \& Durbin (2001) and Karakach \& Wentzell (2007) suggest that the appropriate measurement error model for microarrays is a combination of additive and multiplicative errors.

Since our aim is to understand the direct impact of measurement error, we condition on the data $\mathbf{X}$, fixing the model error. However, recent results raise issues regarding the consistent estimation of the population structure by principal component analysis in the high-dimensional setting. Johnstone \& Lu (2009) among others have shown that eigenvalue and -vector estimates are not asymptotically consistent when $p \gg n$ and they have introduced the asymptotically consistent sparse PCA methodology. Therefore, it remains an open question if the inconsistency may be a more severe problem than measurement error.

\section{Acknowledgement}

This work was supported by the Norwegian Cancer Society, (Project ID 744088).

\section{References}

Buonaccorsi, J. P. (2009). Measurement error: models, methods, and applications. Chapman and Hall, London.

Carroll, R. J., Ruppert, D., Stefanski, L. A. \& Crainiceanu, C. M. (2006). Measurement error in nonlinear models: a modern perspective. CRC Press, London.

Faber, N. M., Buydens, L. M. C. \& Kateman, G. (1993). Standard errors in the eigenvalues of a cross-product matrix: Theory and applications. J. Chemometrics 7, 495-526.

Faber, N. M., Meinders, M. J., Geladi, P., Sjöström, M., Buydens, L. M. C. \& Kateman, G. (1995). Random error bias in principal component analysis. part i. derivation of theoretical predictions. Anal. chimica acta 304, $257-271$.

Fan, J., Lv, J. \& Qi, L. (2011). Sparse high dimensional models in economics. Annual review of economics 3, 291-317.

Ferré, L. (1995). Selection of components in principal component analysis: a comparison of methods. Comput. Statist. Data Anal. 19, 669-682.

Ghazal, G. A. \& Neudecker, H. (2000). On second-order and fourth-order moments of jointly distributed random matrices: a survey. Linear Algebra Appl. 321, 61-93.

Hein, A. M. K., Richardson, S., Causton, H. C., Ambler, G. K. \& Green, P. J. (2005). Bgx: a fully bayesian integrated approach to the analysis of affymetrix genechip data. Biostatistics 6, 349-373. 
Johnstone, I. (2001). On the distribution of the largest eigenvalue in principal components analysis. Ann. Statist. 29, 295-327.

Johnstone, I. \& Lu, A. (2009). On consistency and sparsity for principal components analysis in high dimensions. J. Am. Stat. Assoc. 104, 682-693.

Jolliffe, I. T. (2002). Principal component analysis. Springer, New York.

Kadane, J. B. (1970). Testing overidentifying restrictions when the disturbances are small. J. Am. Stat. Assoc. 65, 182-185.

Karakach, T. K. \& Wentzell, P. D. (2007). Methods for estimating and mitigating errors in spotted, dual-color dna microarrays. Omics: a journal of integrative biology 11, 186-199.

Li, X. \& Xu, R. (2008). High-dimensional data analysis in cancer research. Springer, New York.

Nadler, B. (2008). Finite sample approximation results for principal component analysis: A matrix perturbation approach. Ann. Statist. 36, 2791-2817.

Rao, N. R., Mingo, J. A., Speicher, R. \& Edelman, A. (2008). Statistical eigen-inference from large wishart matrices. Ann. Statist. 36, 2850-2885.

Rocke, D. \& Durbin, B. (2001). A model for measurement error for gene expression arrays. J. Computational Biology 8, 557-569.

Sanguinetti, G., Milo, M., Rattray, M. \& Lawrence, N. D. (2005). Accounting for probe-level noise in principal component analysis of microarray data. Bioinformatics 21, 3748-3754.

Stewart, G. W. (1990). Stochastic perturbation theory. SIAM Rev. 32, 579-610.

Stewart, G. W. \& Sun, J. (1990). Matrix perturbation theory. Academic press, New York.

Turro, E., Bochkina, N., Hein, A. M. K. \& Richardson, S. (2007). Bgx: a bioconductor package for the bayesian integrated analysis of affymetrix genechips. BMC Bioinformatics 8, 439-449.

Wentzell, P. D., Andrews, D. T., Hamilton, D. C., Faber, K. \& Kowalski, B. R. (1997). Maximum likelihood principal component analysis. J. Chemometrics 11, 339-366.

Wentzell, P. D. \& Hou, S. (2012). Exploratory data analysis with noisy measurements. J. Chemometrics 26, 264-281.

Wilkinson, J. H. (1965). The algebraic eigenvalue problem. Clarendon Press, Oxford. 


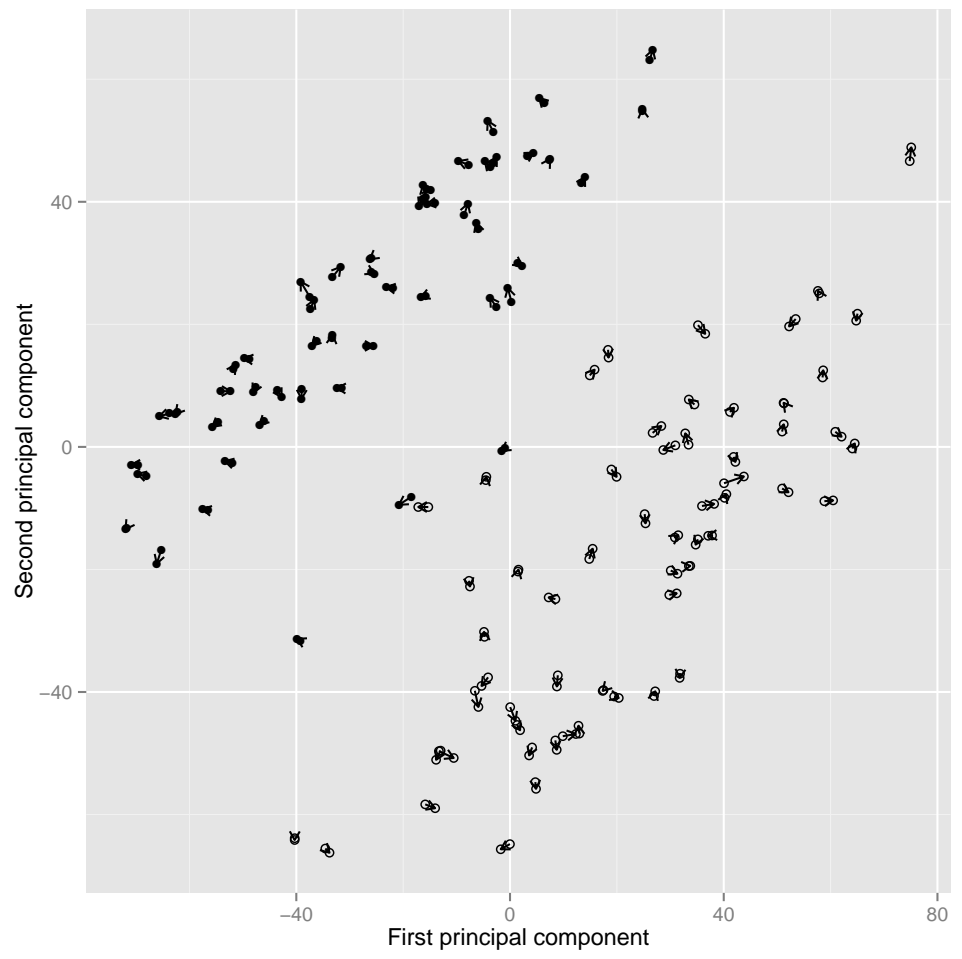

Figure 1: Plot of first and second component scores from original data and data with simulated error based on the estimated error structure. An arrow indicates the change in scores from the original value. Black dots indicate adenocarcinoma tumor tissue and open circles indicate non-tumor tissues. 


\begin{tabular}{c|cc|cc|cc}
\hline Gene annotation & $\mathbf{v}_{1 k}$ & $\Delta \mathbf{v}_{1 k}$ & Bias & St. dev. & $\sigma_{X}^{2}$ & $\sigma_{U}^{2} / \sigma_{X}^{2}$ \\
\hline 214387_x_at & -0.0545 & 0.0008 & 0.000011 & 0.0026 & 8.361 & 0.05 \\
205982_x_at & -0.0539 & -0.0001 & 0.000012 & 0.0025 & 7.830 & 0.05 \\
211735 _x_at & -0.0531 & 0.0038 & 0.000013 & 0.0026 & 8.030 & 0.04 \\
209612_s_at & -0.0528 & 0.0026 & 0.000001 & 0.0026 & 4.839 & 0.15 \\
219230_at & -0.0502 & 0.0048 & -0.000010 & 0.0026 & 4.912 & 0.19 \\
209074_s_at & -0.0501 & 0.0064 & -0.000013 & 0.0025 & 5.276 & 0.18 \\
209613_s_at & -0.0498 & -0.0029 & -0.000006 & 0.0026 & 4.757 & 0.18 \\
203980_at & -0.0496 & 0.0043 & -0.000013 & 0.0026 & 5.397 & 0.18 \\
205200_at & -0.0490 & 0.0006 & -0.000007 & 0.0025 & 4.715 & 0.18 \\
213317_at & -0.0477 & 0.0048 & -0.000004 & 0.0026 & 4.420 & 0.18 \\
204719_at & -0.0476 & 0.0012 & -0.000011 & 0.0025 & 3.755 & 0.26 \\
215454_x_at & -0.0474 & -0.0009 & -0.000001 & 0.0026 & 5.463 & 0.12 \\
209763_at & -0.0469 & 0.0029 & -0.000001 & 0.0025 & 3.659 & 0.22 \\
212713_at & -0.0468 & 0.0012 & -0.000008 & 0.0025 & 3.905 & 0.23 \\
206488_s_at & -0.0463 & -0.0002 & -0.000012 & 0.0025 & 3.773 & 0.27 \\
\hline
\end{tabular}

Table 1: The fifteen genes corresponding to the largest coefficients in absolute value in the first eigenvector in decreasing order, together with the difference induced by the simulated error, theoretical bias, theoretical standard deviations, variable variance and ratio between the variance of measurement error and variance of the variable.

Kristoffer Hellton, Department of Biostatistics, Institute of Basic Medical Sciences, University of Oslo, Postboks 1122 Blindern, 0317 Oslo

E-mail: k.h.hellton@medisin.uio.no 


\section{A Appendix}

\section{A.1 Proof of Lemma 1}

Proof. When $\sigma \rightarrow 0$, we can expand the eigenvalue $\lambda_{W, i}$ around $\lambda_{i}$ and the eigenvector $\mathbf{v}_{W, i}$, up to a scaling, around $\mathbf{v}_{i}$

$$
\begin{aligned}
& \lambda_{W, i}=\lambda_{i}+\sigma \lambda_{1, i}+\sigma^{2} \lambda_{2, i}+O\left(\sigma^{3}\right), \\
& \mathbf{v}_{W, i}=\mathbf{v}_{i}+\sigma \mathbf{v}_{1, i}+\sigma^{2} \mathbf{v}_{2, i}+O\left(\sigma^{3}\right) .
\end{aligned}
$$

The first- and second-order coefficient in the Taylor expansion of the eigenvectors are denoted by $\mathbf{v}_{1, i}$ and $\mathbf{v}_{2, i}$. By inserting these two expressions and $\mathbf{S}_{W}$ in (1) into the eigenequation $\mathbf{S}_{W} \mathbf{v}_{W}=\lambda_{W} \mathbf{v}_{W}$ and collecting the terms with the same power in $\sigma$, the following system of equation is specified

$$
\begin{aligned}
\mathbf{S}_{X} \mathbf{v}_{i} & =\lambda_{i} \mathbf{v}_{i} \\
\mathbf{S}_{X} \mathbf{v}_{1, i}+\Delta \mathbf{S}_{1} \mathbf{v}_{i} & =\lambda_{i} \mathbf{v}_{1, i}+\lambda_{1, i} \mathbf{v}_{i} \\
\mathbf{S}_{X} \mathbf{v}_{2, i}+\Delta \mathbf{S}_{1} \mathbf{v}_{1, i}+\Delta \mathbf{S}_{2} \mathbf{v}_{i} & =\lambda_{i} \mathbf{v}_{2, i}+\lambda_{1, i} \mathbf{v}_{1, i}+\lambda_{2, i} \mathbf{v}_{i} .
\end{aligned}
$$

As the change in an eigenvector must be in terms of the direction, the terms in the Taylor expansions $\mathbf{v}_{1, i}$, $\mathbf{v}_{2, i}$ and the eigenvector $\mathbf{v}_{i}$ must be orthogonal; $\mathbf{v}_{1, i}^{T} \mathbf{v}_{i}=0$ and $\mathbf{v}_{2, i}^{T} \mathbf{v}_{i}=0$. Thus the terms can be written as linear combinations of all the other eigenvectors, such that $\mathbf{v}_{1, i}=\sum_{j \neq i} \alpha_{1, j} \mathbf{v}_{j}$ and $\mathbf{v}_{2, i}=\sum_{j \neq i} \alpha_{2, j} \mathbf{v}_{j}$. First $\lambda_{1, i}$ is found by premultiplying equation (14) by $\mathbf{v}_{i}^{T}$, using the fact that $\mathbf{v}_{i}^{T} \mathbf{v}_{1, i}=0$ and $\mathbf{v}_{i}^{T} \mathbf{v}_{i}=1$

$$
\begin{aligned}
\mathbf{v}_{i}^{T} \mathbf{S}_{X} \mathbf{v}_{1, i}+\mathbf{v}_{i}^{T} \Delta \mathbf{S}_{1} \mathbf{v}_{i} & =\mathbf{v}_{i}^{T} \lambda_{i} \mathbf{v}_{1, i}+\mathbf{v}_{i}^{T} \lambda_{1, i} \mathbf{v}_{i} \\
\lambda_{i} \mathbf{v}_{i}^{T} \mathbf{v}_{1, i}+\mathbf{v}_{i}^{T} \Delta \mathbf{S}_{1} \mathbf{v}_{i} & =\mathbf{v}_{i}^{T} \lambda_{1, i} \mathbf{v}_{i} \\
\lambda_{1, i} & =\mathbf{v}_{i}^{T} \Delta \mathbf{S}_{1} \mathbf{v}_{i}
\end{aligned}
$$

Then $\mathbf{v}_{1, i}$ is found by inserting the linear combination $\mathbf{v}_{1, i}=\sum_{j \neq i} \alpha_{1, j} \mathbf{v}_{j}$ and premultiplying equation (14) by $\mathbf{v}_{j}^{T}$

$$
\begin{aligned}
\mathbf{v}_{j}^{T} \mathbf{S}_{X} \mathbf{v}_{1, i}+\mathbf{v}_{j}^{T} \Delta \mathbf{S}_{1} \mathbf{v}_{i} & =\mathbf{v}_{j}^{T} \lambda_{i} \mathbf{v}_{1, i}+\mathbf{v}_{j}^{T} \lambda_{1, i} \mathbf{v}_{i} \\
\lambda_{j} \alpha_{1, j}+\mathbf{v}_{j}^{T} \Delta \mathbf{S}_{1} \mathbf{v}_{i} & =\lambda_{1, i} \alpha_{1, j} \\
\alpha_{1, j} & =\frac{\mathbf{v}_{j}^{T} \Delta \mathbf{S}_{1} \mathbf{v}_{i}}{\lambda_{i}-\lambda_{j}}
\end{aligned}
$$

Secondly, $\lambda_{2, i}$ is found by premultiplying equation (15) by $\mathbf{v}_{i}^{T}$ :

$$
\begin{aligned}
\mathbf{v}_{i}^{T} \mathbf{S}_{X} \mathbf{v}_{2, i}+\mathbf{v}_{i}^{T} \Delta \mathbf{S}_{1} \mathbf{v}_{1, i}+\mathbf{v}_{i}^{T} \Delta \mathbf{S}_{2} \mathbf{v}_{i} & =\mathbf{v}_{i}^{T} \lambda_{i} \mathbf{v}_{2, i}+\mathbf{v}_{i}^{T} \lambda_{1, i} \mathbf{v}_{1, i}+\mathbf{v}_{i}^{T} \lambda_{2, i} \mathbf{v}_{i} \\
\lambda_{i} \mathbf{v}_{i}^{T} \mathbf{v}_{2, i}+\mathbf{v}_{i}^{T} \Delta \mathbf{S}_{1} \mathbf{v}_{1, i}+\mathbf{v}_{i}^{T} \Delta \mathbf{S}_{2} \mathbf{v}_{i} & =\mathbf{v}_{i}^{T} \lambda_{2, i} \mathbf{v}_{i} \\
\lambda_{2, i} & =\mathbf{v}_{i}^{T} \Delta \mathbf{S}_{2} \mathbf{v}_{i}+\sum_{j \neq i} \frac{\mathbf{v}_{i}^{T} \Delta \mathbf{S}_{1} \mathbf{v}_{j} \mathbf{v}_{j}^{T} \Delta \mathbf{S}_{1} \mathbf{v}_{i}}{\lambda_{i}-\lambda_{j}} .
\end{aligned}
$$


Lastly, the second-order Taylor expansion coefficient for the eigenvector is found by premultiplying equation (15) by $\mathbf{v}_{j}^{T}$ :

$$
\begin{aligned}
\mathbf{v}_{j}^{T} \mathbf{S}_{X} \mathbf{v}_{2, i}+\mathbf{v}_{j}^{T} \Delta \mathbf{S}_{1} \mathbf{v}_{1, i}+\mathbf{v}_{j}^{T} \Delta \mathbf{S}_{2} \mathbf{v}_{i} & =\mathbf{v}_{j}^{T} \lambda_{i} \mathbf{v}_{2, i}+\mathbf{v}_{j}^{T} \lambda_{1, i} \mathbf{v}_{1, i}+\mathbf{v}_{j}^{T} \lambda_{2, i} \mathbf{v}_{i}, \\
\mathbf{v}_{j}^{T} \Delta \mathbf{S}_{1} \mathbf{v}_{1, i}+\mathbf{v}_{j}^{T} \Delta \mathbf{S}_{2} \mathbf{v}_{i}-\lambda_{1, i} \mathbf{v}_{j}^{T} \mathbf{v}_{1, i} & =\lambda_{i} \mathbf{v}_{j}^{T} \mathbf{v}_{2, i}-\lambda_{j} \mathbf{v}_{j}^{T} \mathbf{v}_{2, i}, \\
\mathbf{v}_{j}^{T} \Delta \mathbf{S}_{1} \mathbf{v}_{1, i}+\mathbf{v}_{j}^{T} \Delta \mathbf{S}_{2} \mathbf{v}_{i}-\lambda_{1, i} \alpha_{1, j} & =\left(\lambda_{i}-\lambda_{j}\right) \alpha_{2, j}
\end{aligned}
$$

which, when inserting the expressions for $\lambda_{1, i}$ and $\mathbf{v}_{1, i}$, results in

$$
\alpha_{2, j}=\frac{\mathbf{v}_{j}^{T} \Delta \mathbf{S}_{2} \mathbf{v}_{i}}{\lambda_{i}-\lambda_{j}}+\sum_{k \neq i} \frac{\mathbf{v}_{j}^{T} \Delta \mathbf{S}_{1} \mathbf{v}_{i} \mathbf{v}_{j}^{T} \Delta \mathbf{S}_{1} \mathbf{v}_{k}}{\left(\lambda_{i}-\lambda_{j}\right)\left(\lambda_{i}-\lambda_{k}\right)}-\frac{\mathbf{v}_{j}^{T} \Delta \mathbf{S}_{1} \mathbf{v}_{i} \mathbf{v}_{i}^{T} \Delta \mathbf{S}_{1} \mathbf{v}_{i}}{\left(\lambda_{i}-\lambda_{j}\right)^{2}} .
$$

\section{A.2 Proof of Theorem 1}

Proof. Since the scores are given by $\mathbf{Z}_{W, i}=\mathbf{v}_{W, i}^{T} \mathbf{W}$, the Taylor expansion of $\mathbf{Z}_{W, i}$ around $\mathbf{Z}_{i}=\mathbf{v}_{i}^{T} \mathbf{X}$ is found by combining the Taylor expansion of the eigenvector in expression (3) together with $\mathbf{W}=\mathbf{X}+\sigma \mathbf{U}$. By using the notation of the proof A.1, we obtain

$$
\begin{aligned}
\mathbf{Z}_{W} & =\left(\mathbf{v}_{i}+\sigma \mathbf{v}_{1, i}+\sigma^{2} \mathbf{v}_{2, i}+O\left(\sigma^{3}\right)\right)^{T}(\mathbf{X}+\sigma \mathbf{U}) \\
& =\mathbf{v}_{i}^{T} \mathbf{X}+\sigma\left(\mathbf{v}_{1, i}^{T} \mathbf{X}+\mathbf{v}_{i}^{T} \mathbf{U}\right)+\sigma^{2}\left(\mathbf{v}_{1, i}^{T} \mathbf{U}+\mathbf{v}_{2, i}^{T} \mathbf{X}\right)+O\left(\sigma^{3}\right) .
\end{aligned}
$$

\section{A.3 Second-order moments of random matrices}

For the second-order moments of random matrices, we have the following lemma:

Lemma 2. (Ghazal \& Neudecker, 2000, p.81) For a $p \times n$ matrix $\mathbf{U}=\left[\mathbf{U}_{1}, \ldots, \mathbf{U}_{n}\right]$ where $\mathbf{U}_{r} \sim N(0, \Sigma)$ for $r=1, \ldots, n$, the following is given

$$
\begin{aligned}
\mathbb{E}\left(\mathbf{U A U}^{T}\right) & =\operatorname{tr}(\mathbf{A}) \Sigma, \\
\mathbb{E}\left(\mathbf{U}^{T} \mathbf{A} \mathbf{U}\right) & =\operatorname{tr}(\mathbf{A} \Sigma) I_{n}, \\
\mathbb{E}\left(\mathbf{U}^{T} \mathbf{A} \mathbf{U}^{T}\right) & =\mathbf{A}^{T} \Sigma .
\end{aligned}
$$

Second-order moments of $\Delta \mathbf{S}_{1}=\frac{1}{n} \mathbf{X} \mathbf{U}^{T}+\frac{1}{n} \mathbf{U} \mathbf{X}^{T}$ are found by using Lemma 2.

$$
\begin{gathered}
\mathbb{E}\left(\left[\mathbf{v}_{i}^{T} \Delta \mathbf{S}_{1} \mathbf{v}_{i}\right]^{2} \mid \mathbf{X}\right)=\frac{1}{n^{2}} \mathbb{E}\left(\mathbf{v}_{i}^{T} \mathbf{U} \mathbf{X}^{T} \mathbf{v}_{i}\left(\mathbf{v}_{i}^{T} \mathbf{U} \mathbf{X}^{T} \mathbf{v}_{i}\right)^{T}\right)+\frac{1}{n^{2}} \mathbb{E}\left(\mathbf{v}_{i}^{T} \mathbf{X} \mathbf{U}^{T} \mathbf{v}_{i}\left(\mathbf{v}_{i}^{T} \mathbf{X} \mathbf{U}^{T} \mathbf{v}_{i}\right)^{T} \mid \mathbf{X}\right) \\
+\frac{2}{n^{2}} \mathbb{E}\left(\mathbf{v}_{i}^{T} \mathbf{U} \mathbf{X}^{T} \mathbf{v}_{i}\left(\mathbf{v}_{i}^{T} \mathbf{X} \mathbf{U}^{T} \mathbf{v}_{i}\right)^{T} \mid \mathbf{X}\right) .
\end{gathered}
$$


By using formula (16), the first term yields

$$
\begin{aligned}
\mathbb{E}\left(\mathbf{v}_{i}^{T} \mathbf{U} \mathbf{X}^{T} \mathbf{v}_{i}\left(\mathbf{v}_{i}^{T} \mathbf{U} \mathbf{X}^{T} \mathbf{v}_{i}\right)^{T} \mid \mathbf{X}\right) & =\mathbf{v}_{i}^{T} \mathbb{E}\left(\mathbf{U}\left[\mathbf{X}^{T} \mathbf{v}_{i} \mathbf{v}_{i}^{T} \mathbf{X}\right] \mathbf{U}^{T}\right) \mathbf{v}_{i}=\mathbf{v}_{i}^{T} \operatorname{tr}\left(\mathbf{X}^{T} \mathbf{v}_{i} \mathbf{v}_{i}^{T} \mathbf{X}\right) \Sigma_{U} \mathbf{v}_{i} \\
& =n \mathbf{v}_{i}^{T} \operatorname{tr}\left(\mathbf{v}_{i}^{T} \frac{1}{n} \mathbf{X} \mathbf{X}^{T} \mathbf{v}_{i}\right) \Sigma_{U} \mathbf{v}_{i}=n \lambda_{i} \mathbf{v}_{i}^{T} \Sigma_{U} \mathbf{v}_{i}
\end{aligned}
$$

by formula (17), the second term yields

$$
\begin{gathered}
\mathbb{E}\left(\mathbf{v}_{i}^{T} \mathbf{X} \mathbf{U}^{T} \mathbf{v}_{i}\left(\mathbf{v}_{i}^{T} \mathbf{X} \mathbf{U}^{T} \mathbf{v}_{i}\right)^{T} \mid \mathbf{X}\right)=\mathbf{v}_{i}^{T} \mathbf{X} \mathbb{E}\left(\mathbf{U}^{T}\left[\mathbf{v}_{i} \mathbf{v}_{i}^{T}\right] \mathbf{U}\right) \mathbf{X}^{T} \mathbf{v}_{i}=\mathbf{v}_{i}^{T} \mathbf{X} \operatorname{tr}\left(\mathbf{v}_{i} \mathbf{v}_{i}^{T} \Sigma_{U}\right) I_{n} \mathbf{X}^{T} \mathbf{v}_{i} \\
=n \operatorname{tr}\left(\mathbf{v}_{i}^{T} \Sigma_{U} \mathbf{v}_{i}\right) \mathbf{v}_{i}^{T} \frac{1}{n} \mathbf{X} \mathbf{X}^{T} \mathbf{v}_{i}=n \lambda_{i} \mathbf{v}_{i}^{T} \Sigma_{U} \mathbf{v}_{i},
\end{gathered}
$$

and by formula (18), the third term yields

$$
\begin{aligned}
\mathbb{E}\left(\mathbf{v}_{i}^{T} \mathbf{X} \mathbf{U}^{T} \mathbf{v}_{i}\left(\mathbf{v}_{i}^{T} \mathbf{U} \mathbf{X}^{T} \mathbf{v}_{i}\right)^{T} \mid \mathbf{X}\right) & =\mathbf{v}_{i}^{T} \mathbf{X} \mathbb{E}\left(\mathbf{U}^{T}\left[\mathbf{v}_{i} \mathbf{v}_{i}^{T} \mathbf{X}^{T}\right] \mathbf{U}^{T}\right) \mathbf{v}_{i}=\mathbf{v}_{i}^{T} \mathbf{X} \Sigma_{U}\left[\mathbf{v}_{i} \mathbf{v}_{i}^{T} \mathbf{X}^{T}\right]^{T} \mathbf{v}_{i} \\
=n \mathbf{v}_{i}^{T} \frac{1}{n} \mathbf{X} \mathbf{X}^{T} \mathbf{v}_{i} \mathbf{v}^{T} \Sigma_{U} \mathbf{v}_{i} & =n \lambda_{i} \mathbf{v}_{i}^{T} \Sigma_{U} \mathbf{v}_{i}
\end{aligned}
$$

such that

$$
\mathbb{E}\left(\left[\mathbf{v}_{i}^{T} \Delta \mathbf{S}_{1} \mathbf{v}_{i}\right]^{2} \mid \mathbf{X}\right)=\frac{4 \lambda_{i}}{n} \mathbf{v}^{T} \Sigma_{U} \mathbf{v} .
$$

When the eigenvector indexes are different, the second-order moment is given by

$$
\begin{gathered}
\mathbb{E}\left(\left[\mathbf{v}_{j}^{T} \Delta \mathbf{S}_{1} \mathbf{v}_{i}\right]^{2} \mid \mathbf{X}\right)=\frac{1}{n^{2}} \mathbb{E}\left(\mathbf{v}_{j}^{T} \mathbf{U} \mathbf{X}^{T} \mathbf{v}_{i}\left(\mathbf{v}_{j}^{T} \mathbf{U} \mathbf{X}^{T} \mathbf{v}_{i}\right)^{T} \mid \mathbf{X}\right)+\frac{1}{n^{2}} \mathbb{E}\left(\mathbf{v}_{j}^{T} \mathbf{X} \mathbf{U}^{T} \mathbf{v}_{i}\left(\mathbf{v}_{j}^{T} \mathbf{X} \mathbf{U}^{T} \mathbf{v}_{i}\right)^{T} \mid \mathbf{X}\right) \\
+\frac{2}{n^{2}} \mathbb{E}\left(\mathbf{v}_{j}^{T} \mathbf{X} \mathbf{U}^{T} \mathbf{v}_{i}\left(\mathbf{v}_{j}^{T} \mathbf{U} \mathbf{X}^{T} \mathbf{v}_{i}\right)^{T} \mid \mathbf{X}\right) .
\end{gathered}
$$

The first term yields

$$
\begin{aligned}
\mathbb{E}\left(\mathbf{v}_{j}^{T} \mathbf{U} \mathbf{X}^{T} \mathbf{v}_{i}\left(\mathbf{v}_{j}^{T} \mathbf{U} \mathbf{X}^{T} \mathbf{v}_{i}\right)^{T} \mid \mathbf{X}\right) & =\mathbf{v}_{j}^{T} \mathbb{E}\left(\mathbf{U}\left[\mathbf{X}^{T} \mathbf{v}_{i} \mathbf{v}_{i}^{T} \mathbf{X}\right] \mathbf{U}^{T}\right) \mathbf{v}_{j}=\mathbf{v}_{j}^{T} \operatorname{tr}\left(\mathbf{X}^{T} \mathbf{v}_{i} \mathbf{v}_{i}^{T} \mathbf{X}\right) \Sigma_{U} \mathbf{v}_{j} \\
& =n \operatorname{tr}\left(\mathbf{v}_{i}^{T} \frac{1}{n} \mathbf{X} \mathbf{X}^{T} \mathbf{v}_{i}\right) \mathbf{v}_{j}^{T} \Sigma_{U} \mathbf{v}_{j}=n \lambda_{i} \mathbf{v}_{j}^{T} \Sigma_{U} \mathbf{v}_{j},
\end{aligned}
$$

the second term yields

$$
\begin{aligned}
\mathbb{E}\left(\mathbf{v}_{j}^{T} \mathbf{X} \mathbf{U}^{T} \mathbf{v}_{i}\left(\mathbf{v}_{j}^{T} \mathbf{U} \mathbf{X}^{T} \mathbf{v}_{i}\right)^{T} \mid \mathbf{X}\right) & =\mathbf{v}_{j}^{T} \mathbf{X} \mathbb{E}\left(\mathbf{U}^{T}\left[\mathbf{v}_{i} \mathbf{v}_{i}^{T}\right] \mathbf{U}\right) \mathbf{X}^{T} \mathbf{v}_{j}=\mathbf{v}_{j}^{T} \mathbf{X} \operatorname{tr}\left(\mathbf{v}_{i} \mathbf{v}_{i}^{T} \Sigma_{U}\right) I_{n} \mathbf{X}^{T} \mathbf{v}_{j}, \\
& =n \operatorname{tr}\left(\mathbf{v}_{i}^{T} \Sigma_{U} \mathbf{v}_{i}\right) \mathbf{v}_{j}^{T} \frac{1}{n} \mathbf{X} \mathbf{X}^{T} \mathbf{v}_{j}=n \lambda_{j} \mathbf{v}_{i}^{T} \Sigma_{U} \mathbf{v}_{i},
\end{aligned}
$$

and the third term yields

$$
\begin{aligned}
\mathbb{E}\left(\mathbf{v}_{j}^{T} \mathbf{U} \mathbf{X}^{T} \mathbf{v}_{i} \mathbf{v}_{i}^{T} \mathbf{U} \mathbf{X}^{T} \mathbf{v}_{j} \mid \mathbf{X}\right)=\mathbf{v}_{j}^{T} \mathbb{E}\left(\mathbf{U}\left[\mathbf{X}^{T} \mathbf{v}_{i} \mathbf{v}_{i}^{T} \mathbf{U}\right]\right) \mathbf{X}^{T} \mathbf{v}_{j}, \\
=\mathbf{v}_{j}^{T} \Sigma_{U}\left[\mathbf{X}^{T} \mathbf{v}_{i} \mathbf{v}_{i}^{T}\right]^{T} \mathbf{X}^{T} \mathbf{v}_{j},=\mathbf{v}_{j}^{T} \Sigma_{U} \mathbf{v}_{i} \mathbf{v}_{i}^{T} \mathbf{X} \mathbf{X}^{T} \mathbf{v}_{j}=0,
\end{aligned}
$$

due to the fact that the $i$ th and $j$ th score vectors are uncorrelated by definition, $\operatorname{Cov}\left(\mathbf{v}_{i}^{T} \mathbf{X}, \mathbf{v}_{j}^{T} \mathbf{X}\right)=0$. Thus, 
the second-order moment is given by

$$
\mathbb{E}\left(\left[\mathbf{v}_{j}^{T} \Delta \mathbf{S}_{1} \mathbf{v}_{i}\right]^{2} \mid \mathbf{X}\right)=\frac{\lambda_{j}}{n} \mathbf{v}_{i}^{T} \Sigma_{U} \mathbf{v}_{i}+\frac{\lambda_{i}}{n} \mathbf{v}_{j}^{T} \Sigma_{U} \mathbf{v}_{j} .
$$

$\operatorname{For} \mathbb{E}\left(\mathbf{v}_{j}^{T} \Delta \mathbf{S}_{1} \mathbf{v}_{i} \mathbf{v}_{j}^{T} \Delta \mathbf{S}_{1} \mathbf{v}_{k}\right)$ for $k \neq j$, we obtain

$$
\mathbb{E}\left(\mathbf{v}_{j}^{T} \mathbf{U} \mathbf{X}^{T} \mathbf{v}_{i}\left(\mathbf{v}_{j}^{T} \mathbf{U} \mathbf{X}^{T} \mathbf{v}_{k}\right)^{T} \mid \mathbf{X}\right)=\frac{\lambda_{j}}{n} \mathbf{v}_{k}^{T} \Sigma_{U} \mathbf{v}_{i}
$$

while all other combinations yield zero, such that

$$
\mathbb{E}\left(\mathbf{v}_{j}^{T} \Delta \mathbf{S}_{1} \mathbf{v}_{i} \mathbf{v}_{j}^{T} \Delta \mathbf{S}_{1} \mathbf{v}_{k} \mid \mathbf{X}\right)=\frac{\lambda_{j}}{n} \mathbf{v}_{k}^{T} \Sigma_{U} \mathbf{v}_{i}, \quad k \neq j .
$$

To calculate the moment $\mathbb{E}\left(\mathbf{v}_{j}^{T} \Delta \mathbf{S}_{1} \mathbf{v}_{i} \mathbf{v}_{i}^{T} \Delta \mathbf{S}_{1} \mathbf{v}_{i} \mid \mathbf{X}\right)$, we first obtain

$$
\begin{aligned}
& \mathbb{E}\left(\mathbf{v}_{j}^{T} \mathbf{U} \mathbf{X}^{T} \mathbf{v}_{i} \mathbf{v}_{i}^{T} \mathbf{X} \mathbf{U}^{T} \mathbf{v}_{i}\right)=n \lambda_{i} \mathbf{v}_{j}^{T} \Sigma_{U} \mathbf{v}_{i}, \\
& \mathbb{E}\left(\mathbf{v}_{j}^{T} \mathbf{U} \mathbf{X}^{T} \mathbf{v}_{i} \mathbf{v}_{i}^{T} \mathbf{U} \mathbf{X}^{T} \mathbf{v}_{i}\right)=n \lambda_{i} \mathbf{v}_{j}^{T} \Sigma_{U} \mathbf{v}_{i},
\end{aligned}
$$

while the two other combinations yield zero, such that

$$
\mathbb{E}\left(\mathbf{v}_{j}^{T} \Delta \mathbf{S}_{1} \mathbf{v}_{i} \mathbf{v}_{i}^{T} \Delta \mathbf{S}_{1} \mathbf{v}_{i} \mid \mathbf{X}\right)=\frac{2 \lambda_{i}}{n} \mathbf{v}_{j}^{T} \Sigma_{U} \mathbf{v}_{i}
$$

In addition, the formulas in Lemma 2 give

$$
\begin{aligned}
& \mathbb{E}\left(\mathbf{v}_{j}^{T} \Delta \mathbf{S}_{1} \mathbf{v}_{i} \mathbf{v}_{j}^{T} \mathbf{U} \mid \mathbf{X}\right)=\mathbf{v}_{j}^{T} \Sigma_{U} \mathbf{v}_{i} \mathbf{v}_{j}^{T} \mathbf{X}+\mathbf{v}_{j}^{T} \Sigma_{U} \mathbf{v}_{j} \mathbf{v}_{i}^{T} \mathbf{X}, \\
& \mathbb{E}\left(\mathbf{v}_{j}^{T} \Delta \mathbf{S}_{1} \mathbf{v}_{i} \mathbf{v}_{i}^{T} \mathbf{U} \mid \mathbf{X}\right)=\mathbf{v}_{j}^{T} \Sigma_{U} \mathbf{v}_{i} \mathbf{v}_{i}^{T} \mathbf{X}+\mathbf{v}_{i}^{T} \Sigma_{U} \mathbf{v}_{i} \mathbf{v}_{j}^{T} \mathbf{X} .
\end{aligned}
$$

\section{A.4 Proof of Theorem 2}

Proof. The bias in the $i$ th eigenvalue is found by moving $\lambda_{i}$ in expression (2) to the other side and take the expectation

$$
\mathbb{E}\left(\Delta \lambda_{i} \mid \mathbf{X}\right)=\sigma \mathbf{v}_{i}^{T} \mathbb{E}\left(\Delta \mathbf{S}_{1}\right) \mathbf{v}_{i}+\sigma^{2} \mathbf{v}_{i}^{T} \mathbb{E}\left(\Delta \mathbf{S}_{2}\right) \mathbf{v}_{i}+\sigma^{2} \sum_{j \neq i} \frac{\mathbb{E}\left(\mathbf{v}_{i}^{T} \Delta \mathbf{S}_{1} \mathbf{v}_{j} \mathbf{v}_{j}^{T} \Delta \mathbf{S}_{1} \mathbf{v}_{i}\right)}{\lambda_{i}-\lambda_{j}}+O\left(\sigma^{3}\right) .
$$

When the error $\mathbf{U}_{r}$ is iid $\mathbf{U}_{r} \sim N\left(0, \Sigma_{U}\right)$, it follows that $\mathbb{E}\left(\Delta \mathbf{S}_{1}\right)=\mathbf{0}$ and $\mathbb{E}\left(\Delta \mathbf{S}_{2}\right)=\Sigma_{U}$. By inserting these expectations together with the expression (21), derived in the Appendix A.3, we obtain the result.

The bias in the $i$ th eigenvector is found by moving $\mathbf{v}_{i}$ in expression (3) to the other side and take the expectation

$$
\begin{aligned}
\mathbb{E}\left(\Delta \mathbf{v}_{i} \mid \mathbf{X}\right) & =\sigma \sum_{j \neq i} \frac{\mathbf{v}_{j}^{T} \mathbb{E}\left(\Delta \mathbf{S}_{1}\right) \mathbf{v}_{i}}{\lambda_{i}-\lambda_{j}} \mathbf{v}_{j}+\sigma^{2} \sum_{j \neq i} \frac{\mathbf{v}_{j}^{T} \mathbb{E}\left(\Delta \mathbf{S}_{2}\right) \mathbf{v}_{i}}{\lambda_{i}-\lambda_{j}} \mathbf{v}_{j} \\
& +\sigma^{2} \sum_{j \neq i} \sum_{k \neq i} \frac{\mathbb{E}\left(\mathbf{v}_{j}^{T} \Delta \mathbf{S}_{1} \mathbf{v}_{i} \mathbf{v}_{j}^{T} \Delta \mathbf{S}_{1} \mathbf{v}_{k}\right)}{\left(\lambda_{i}-\lambda_{j}\right)\left(\lambda_{i}-\lambda_{k}\right)} \mathbf{v}_{j}-\sigma^{2} \sum_{j \neq i} \frac{\mathbb{E}\left(\mathbf{v}_{j}^{T} \Delta \mathbf{S}_{1} \mathbf{v}_{i} \mathbf{v}_{i}^{T} \Delta \mathbf{S}_{1} \mathbf{v}_{i}\right)}{\left(\lambda_{i}-\lambda_{j}\right)^{2}} \mathbf{v}_{j}+O\left(\sigma^{3}\right) .
\end{aligned}
$$


We insert $\mathbb{E}\left(\Delta \mathbf{S}_{1}\right)=\mathbf{0}$ and $\mathbb{E}\left(\Delta \mathbf{S}_{2}\right)=\Sigma_{U}$ and the expressions (21) and (22) derived in the Appendix A.3 and get the result by rearranging the two last terms.

The variance of the change in the $i$ th eigenvalue is given by

$$
\operatorname{Var}\left(\Delta \lambda_{i} \mid \mathbf{X}\right)=\sigma^{2} \operatorname{Var}\left(\mathbf{v}_{i}^{T} \Delta \mathbf{S}_{1} \mathbf{v}_{i}\right)+O\left(\sigma^{3}\right)=\sigma^{2} \mathbb{E}\left(\left[\mathbf{v}_{i}^{T} \Delta \mathbf{S}_{1} \mathbf{v}_{i}\right]^{2}\right)-\sigma^{2}\left(\mathbf{v}_{i}^{T} \mathbb{E} \Delta \mathbf{S}_{1} \mathbf{v}_{i}\right)^{2}+O\left(\sigma^{3}\right) .
$$

As $\mathbb{E}\left(\Delta \mathbf{S}_{1}\right)=\mathbf{0}$, the variance is equal to the second-order moment in equation (20), such that

$$
\operatorname{Var}\left(\Delta \lambda_{i} \mid \mathbf{X}\right)=\frac{4 \sigma^{2} \lambda_{i}}{n} \mathbf{v}_{i}^{T} \Sigma_{U} \mathbf{v}_{i}+O\left(\sigma^{3}\right)
$$

The variance of the change in the $k$ th coordinate of the $i$ th eigenvector is given by

$$
\begin{aligned}
\operatorname{Var}\left(\Delta \mathbf{v}_{i k} \mid \mathbf{X}\right) & =\sigma^{2} \operatorname{Var}\left(\sum_{j \neq i} \frac{\mathbf{v}_{j}^{T} \Delta \mathbf{S}_{1} \mathbf{v}_{i}}{\lambda_{i}-\lambda_{j}} \mathbf{v}_{j k}\right)+O\left(\sigma^{3}\right), \\
& =\sigma^{2} \sum_{j \neq i} \frac{\operatorname{Var}\left(\mathbf{v}_{j}^{T} \Delta \mathbf{S}_{1} \mathbf{v}_{i}\right)}{\left(\lambda_{i}-\lambda_{j}\right)^{2}} \mathbf{v}_{j k}^{2}+2 \sigma^{2} \sum_{j, l \neq i, j<l} \frac{\operatorname{Cov}\left(\mathbf{v}_{j}^{T} \Delta \mathbf{S}_{1} \mathbf{v}_{i}, \mathbf{v}_{l}^{T} \Delta \mathbf{S}_{1} \mathbf{v}_{i}\right)}{\left(\lambda_{i}-\lambda_{j}\right)\left(\lambda_{i}-\lambda_{l}\right)} \mathbf{v}_{j k} \mathbf{v}_{l k}+O\left(\sigma^{3}\right) .
\end{aligned}
$$

As $\mathbb{E}\left(\Delta \mathbf{S}_{1}\right)=0$, the variance and covariance expressions are given by the second-order moments

$$
\begin{aligned}
\operatorname{Var}\left(\mathbf{v}_{j}^{T} \Delta \mathbf{S}_{1} \mathbf{v}_{i}\right) & =\mathbb{E}\left(\left[\mathbf{v}_{j}^{T} \Delta \mathbf{S}_{1} \mathbf{v}_{i}\right]^{2}\right) \\
\operatorname{Cov}\left(\mathbf{v}_{j}^{T} \Delta \mathbf{S}_{1} \mathbf{v}_{i}, \mathbf{v}_{l}^{T} \Delta \mathbf{S}_{1} \mathbf{v}_{i}\right) & =\mathbb{E}\left(\mathbf{v}_{j}^{T} \Delta \mathbf{S}_{1} \mathbf{v}_{i} \mathbf{v}_{l}^{T} \Delta \mathbf{S}_{1} \mathbf{v}_{i}\right)
\end{aligned}
$$

which are given by expression (21) and (22).

\section{A.5 Proof of Theorem 3}

Proof. The bias in the scores is found by moving $\mathbf{Z}_{i}$ in expression (4) to the other side and take the expectation

$$
\begin{aligned}
\mathbb{E}\left(\Delta \mathbf{Z}_{i} \mid \mathbf{X}\right) & =\sigma \sum_{j \neq i} \frac{\mathbb{E}\left(\mathbf{v}_{j}^{T} \Delta \mathbf{S}_{1} \mathbf{v}_{i}\right)}{\lambda_{i}-\lambda_{j}} \mathbf{v}_{j}^{T} \mathbf{X}+\sigma \mathbf{v}_{i}^{T} \mathbb{E} \mathbf{U}+\sigma^{2} \sum_{j \neq i} \frac{\mathbb{E}\left(\mathbf{v}_{j}^{T} \Delta \mathbf{S}_{1} \mathbf{v}_{i} \mathbf{v}_{j}^{T} \mathbf{U}\right)}{\lambda_{i}-\lambda_{j}}+\sigma^{2} \sum_{j \neq i} \frac{\mathbb{E}\left(\mathbf{v}_{j}^{T} \Delta \mathbf{S}_{2} \mathbf{v}_{i}\right)}{\lambda_{i}-\lambda_{j}} \mathbf{v}_{j}^{T} \mathbf{X} \\
& +\sigma^{2} \sum_{j \neq i} \sum_{k \neq i} \frac{\mathbb{E}\left(\mathbf{v}_{j}^{T} \Delta \mathbf{S}_{1} \mathbf{v}_{i} \mathbf{v}_{j}^{T} \Delta \mathbf{S}_{1} \mathbf{v}_{k}\right)}{\left(\lambda_{i}-\lambda_{j}\right)\left(\lambda_{i}-\lambda_{k}\right)} \mathbf{v}_{j}^{T} \mathbf{X}-\sigma^{2} \sum_{j \neq i} \frac{\mathbb{E}\left(\mathbf{v}_{j}^{T} \Delta \mathbf{S}_{1} \mathbf{v}_{i} \mathbf{v}_{i}^{T} \Delta \mathbf{S}_{1} \mathbf{v}_{i}\right)}{\left(\lambda_{i}-\lambda_{j}\right)^{2}} \mathbf{v}_{j}^{T} \mathbf{X}+O\left(\sigma^{3}\right) .
\end{aligned}
$$

Apart from $\mathbb{E}\left(\Delta \mathbf{S}_{1}\right)=\mathbf{0}, \mathbb{E}\left(\Delta \mathbf{S}_{2}\right)=\Sigma_{U}$ and $\mathbb{E}(\mathbf{U})=\mathbf{0}$, the expectations are given by the expressions in (22), (23) and (24). The result is obtained by inserting all expectations. 
The variance of the change in the $k$ th score of the $i$ th component is given by

$$
\begin{aligned}
\operatorname{Var}\left(\Delta \mathbf{Z}_{i k} \mid \mathbf{X}\right) & =\sigma^{2} \operatorname{Var}\left(\sum_{j \neq i} \frac{\mathbf{v}_{j}^{T} \Delta \mathbf{S}_{1} \mathbf{v}_{i}}{\lambda_{i}-\lambda_{j}} \mathbf{v}_{j}^{T} \mathbf{X}_{k}+\mathbf{v}_{i}^{T} \mathbf{U}_{k} \mid \mathbf{X}\right)+O\left(\sigma^{3}\right) \\
& =\sigma^{2} \operatorname{Var}\left(\sum_{j \neq i} \frac{\mathbf{v}_{j}^{T} \Delta \mathbf{S}_{1} \mathbf{v}_{i}}{\lambda_{i}-\lambda_{j}} \mathbf{v}_{j}^{T} \mathbf{X}_{k} \mid \mathbf{X}\right)+\sigma^{2} \operatorname{Var}\left(\mathbf{v}_{i}^{T} \mathbf{U}_{k} \mid \mathbf{X}\right) \\
& +2 \sigma^{2} \operatorname{Cov}\left(\sum_{j \neq i} \frac{\mathbf{v}_{j}^{T} \Delta \mathbf{S}_{1} \mathbf{v}_{i}}{\lambda_{i}-\lambda_{j}} \mathbf{v}_{j}^{T} \mathbf{X}_{k}, \mathbf{v}_{i}^{T} \mathbf{U}_{k} \mid \mathbf{X}\right)+O\left(\sigma^{3}\right) .
\end{aligned}
$$

The first term is directly given by the variance of the eigenvector

$$
\begin{aligned}
\operatorname{Var}\left(\sum_{j \neq i} \frac{\mathbf{v}_{j}^{T} \Delta \mathbf{S}_{1} \mathbf{v}_{i}}{\lambda_{i}-\lambda_{j}} \mathbf{v}_{j}^{T} \mathbf{X}_{k} \mid \mathbf{X}\right) & =\sum_{j \neq i} \frac{\operatorname{Var}\left(\mathbf{v}_{j}^{T} \Delta \mathbf{S}_{1} \mathbf{v}_{i}\right)}{\left(\lambda_{i}-\lambda_{j}\right)^{2}}\left(\mathbf{v}_{j}^{T} \mathbf{X}_{k}\right)^{2}+2 \sum_{j, l \neq i, j<l} \frac{\operatorname{Cov}\left(\mathbf{v}_{j}^{T} \Delta \mathbf{S}_{1} \mathbf{v}_{i}, \mathbf{v}_{l}^{T} \Delta \mathbf{S}_{1} \mathbf{v}_{i}\right)}{\left(\lambda_{i}-\lambda_{j}\right)\left(\lambda_{i}-\lambda_{l}\right)} \mathbf{v}_{j}^{T} \mathbf{X}_{k} \mathbf{v}_{l}^{T} \mathbf{X}_{k}, \\
& =\sum_{j \neq i} \frac{\lambda_{j} \mathbf{v}_{i}^{T} \Sigma_{U} \mathbf{v}_{i}+\lambda_{i} \mathbf{v}_{j}^{T} \Sigma_{U} \mathbf{v}_{j}}{n\left(\lambda_{i}-\lambda_{j}\right)^{2}}\left(\mathbf{v}_{j}^{T} \mathbf{X}_{k}\right)^{2}+\sum_{j, l \neq i, j<l} \frac{2 \lambda_{i} \mathbf{v}_{j}^{T} \Sigma_{U} \mathbf{v}_{l}}{n\left(\lambda_{i}-\lambda_{j}\right)\left(\lambda_{i}-\lambda_{l}\right)} \mathbf{v}_{j}^{T} \mathbf{X}_{k} \mathbf{v}_{l}^{T} \mathbf{X}_{k} .
\end{aligned}
$$

The second term, the direct contribution from the error, is given

$$
\operatorname{Var}\left(\mathbf{v}_{i}^{T} \mathbf{U}_{k} \mid \mathbf{X}\right)=\mathbf{v}_{i}^{T} \Sigma_{U} \mathbf{v}_{i}
$$

The last term is given by the interaction between the error and the eigenvector as $\mathbb{E} \mathbf{U}=\mathbf{0}$

$$
\operatorname{Cov}\left(\sum_{j \neq i} \frac{\mathbf{v}_{j}^{T} \Delta \mathbf{S}_{1} \mathbf{v}_{i}}{\lambda_{i}-\lambda_{j}} \mathbf{v}_{j}^{T} \mathbf{X}_{k}, \mathbf{v}_{i}^{T} \mathbf{U}_{k} \mid \mathbf{X}\right)=\sum_{j \neq i} \frac{\mathbb{E}\left(\mathbf{v}_{j}^{T} \Delta \mathbf{S}_{1} \mathbf{v}_{i} \mathbf{v}_{i}^{T} \mathbf{U}_{k}\right)}{\lambda_{i}-\lambda_{j}} \mathbf{v}_{j} \mathbf{X}_{k},
$$

where the expectation is given by the $k$ th coefficient in expression (25):

$$
\mathbb{E}\left(\mathbf{v}_{j}^{T} \Delta \mathbf{S}_{1} \mathbf{v}_{i} \mathbf{v}_{i}^{T} \mathbf{U}_{k} \mid \mathbf{X}\right)=\frac{1}{n} \mathbf{v}_{j}^{T} \Sigma_{U} \mathbf{v}_{i} \mathbf{v}_{i}^{T} \mathbf{X}_{k}+\frac{1}{n} \mathbf{v}_{i}^{T} \Sigma_{U} \mathbf{v}_{i} \mathbf{v}_{j}^{T} \mathbf{X}_{k}
$$

The result is given by combining the three terms. 